\title{
SUPPLEMENT TO: NONPARAMETRIC ESTIMATION OF PREGNANCY OUTCOME PROBABILITIES
}

\author{
By Sarah Friedrich * , Jan Beyersmann*, Ursula Winterfeld ${ }^{\dagger}$, \\ Martin Schumacher ${ }^{\ddagger}$ And Arthur Allignol* \\ Institute of Statistics, Ulm University, Germany*, \\ STIS and Division of Clinical Pharmacology and Toxicology, University \\ Hospital Lausanne, Switzerland ${ }^{\dagger}$ \\ and Institute of Medical Biometry and Medical Informatics, University \\ Medical Center Freiburg, Germany ${ }^{\ddagger}$
}

\begin{abstract}
In this supplementary material we consider some additional theoretical details as well as the classical survival case and a modified Kaplan-Meier estimator. Furthermore, we provide some additional simulation results as well as a simulation scenario with less pronounced truncation.
\end{abstract}

\section{Additional theoretical results.}

7.1. The connection between the competing risks setting and the statin study. Our interest lies in estimating the risk of spontaneous abortion after drug exposure during pregnancy. In the statin study, pregnancy outcomes of women, who were exposed to statins and contacted a TIS, were compared to a control group.

The analysis takes place in a competing risks setting, since a pregnancy may end in a spontaneous or an induced abortion or a live birth. Furthermore, the data are left-truncated since women usually become aware of a pregnancy and contact a TIS several weeks after conception. A model for this setting is given in Figure 6.

However, events like spontaneous or induced abortion often happen early during pregnancy, when only a small number of women are under observation. This leads to problems concerning estimation and can cause unreliable estimates. Due to the cumulative nature of the estimators these problems propagate.

7.2. The new filtration. Practically speaking, Assumption 3.1 implies that the knowledge of whether or not more patients enter the study later does not change the intensity of having an event for a single individual. This assumption is stricter than conditioning on the usual filtration. An example is given in Remark 7.1. 


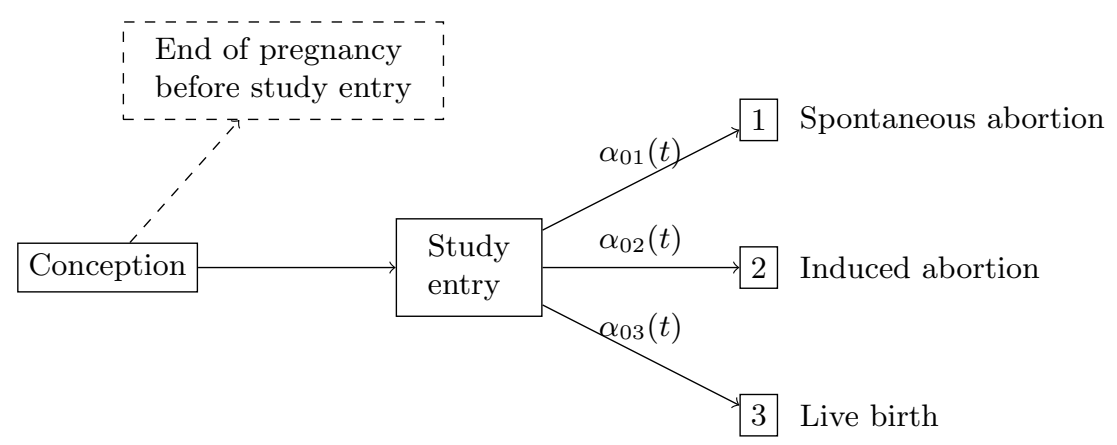

Fig 6: Competing risks model for pregnancy data and observation process.

REMARK 7.1. It is possible to construct examples where the new filtration does change the intensity of having an event. Consider the following - rather artificial - example: Assume we have a study, in which we only ever include one person at a time and then follow this individual until we observe an event (i.e., no censoring). After observing an event, we will include another individual in the study (assuming that there will always be enough persons available for inclusion). When conditioning on the usual filtration, the intensity of having an event does not change for our individual under study, assuming independence across individuals. However, if we additionally condition on the total number of individuals under study being one, for example, this implies that our individual under study will never have an event (otherwise more individuals would be included), thus reducing the intensity of experiencing an event to 0. Therefore, the assumption that conditioning on $\mathcal{F}(t)$ does not change the intensities of the counting processes is stricter than conditioning on the usual filtration.

8. The classical survival case and a modified Kaplan-Meier estimator. The results from Section 3.1 also hold true for the classical survival case, where we consider time until death. The counting process of observed events is given by

$$
N_{m(n)}(t)=\sum_{i=1}^{m(n)} \mathbb{1}\left(L_{i}<\tilde{T}_{i} \leq t, \delta_{i}=1\right)
$$

and the at-risk process by

$$
Y_{m(n)}(t)=\sum_{i=1}^{m(n)} \mathbb{1}\left(L_{i}<t \leq \tilde{T}_{i}\right) .
$$


The modified Nelson-Aalen estimator is

$$
\hat{A}(t)=\sum_{s \leq t} \frac{\Delta N_{m(n)}(s)}{Y_{m(n)}(s)} \mathbb{1}\left(Y_{m(n)}(s) \geq c n^{\gamma}\right) .
$$

Using (8.1), we can now easily define a modified version of the KaplanMeier estimator by using product integration (Gill and Johansen, 1990):

$$
\hat{S}(t)=\prod_{(0, t]}(1-d \hat{A})=\prod_{s \leq t}\left(1-\frac{\Delta N_{m(n)}(s)}{Y_{m(n)}(s)} \mathbb{1}\left(Y_{m(n)}(s) \geq c n^{\gamma}\right)\right),
$$

where the product is over all observed, unique event times $s, s \leq t$.

From Theorem 3.3 we know that $\sqrt{n}(\hat{A}-A)$ converges weakly to a Gaussian martingale $W$ with covariance function

$$
\sigma^{2}(t)=\int_{0}^{t} \frac{\alpha(s)}{y(s)} d s .
$$

Since the survival function $S(A)=\pi(1-d A)$ is a functional of the integrated hazard $A$, we can use the functional delta method to derive asymptotic properties for the Kaplan-Meier estimator:

The mapping $S$ is compactly differentiable with derivative

$$
(d S(A) \cdot h)(s)=-\int_{0}^{s} S(u-)\left(\frac{S(s)}{S(u)}\right) d h(u)=-S(s) h(s)
$$

with $h \in D[0, t]$ (Andersen et al., 1993). The functional delta method therefore gives that

$$
\sqrt{n}(\hat{S}-S) \stackrel{\mathcal{D}}{\rightarrow}-S \cdot W=-(1-F) \cdot W .
$$

These results are the same as the ones found by Lai and Ying (1991, Theorem $5)$. Note that the asymptotic distribution is the same as for the standard Kaplan-Meier estimator.

\section{Further simulation results.}

9.1. Small sample behavior. Here, we have included further simulation results of the small sample scenario described in Section 4.2. Figure 7 displays the mean Aalen-Johansen estimator and its modified version for $c=1$ and $\gamma=0.25$. It turns out that the modified estimator has the larger absolute bias on average and a smaller variation. This is due to the fact that 
the modified estimator avoids 'outliers', see Figure 8, which displays randomly chosen CIFs estimated by the usual Aalen-Johansen estimator. We see a large amount of these reaching implausibly high levels at the plateau, because an event happened early, adding to the variability of the usual Aalen-Johansen estimator. Figure 9 illustrates that the modified estimator makes sense only for a 'meaningful' choice of $\gamma$ and $c$.

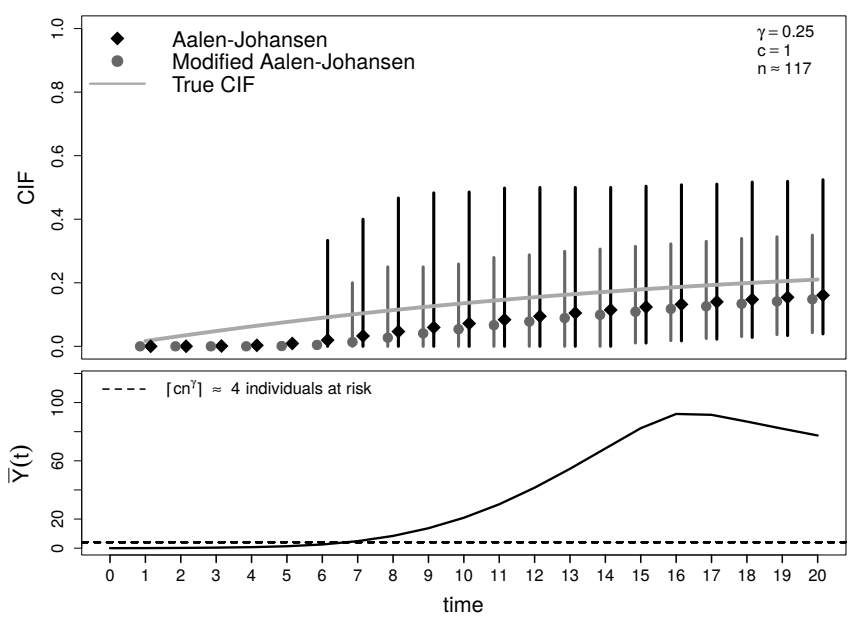

Fig 7: Results of 10000 simulation runs in the small sample scenario: On average $n=117$ out of 200 simulated individuals enter the study. Displayed are the mean Aalen-Johansen estimator and its modified version along with empirical $95 \%$ CIs. The lower plot shows the average number of individuals at risk over the course of time. 


\section{SUPPLEMENT TO: NONPARAMETRIC ESTIMATION OF PREGNANCY OUTCOMES}

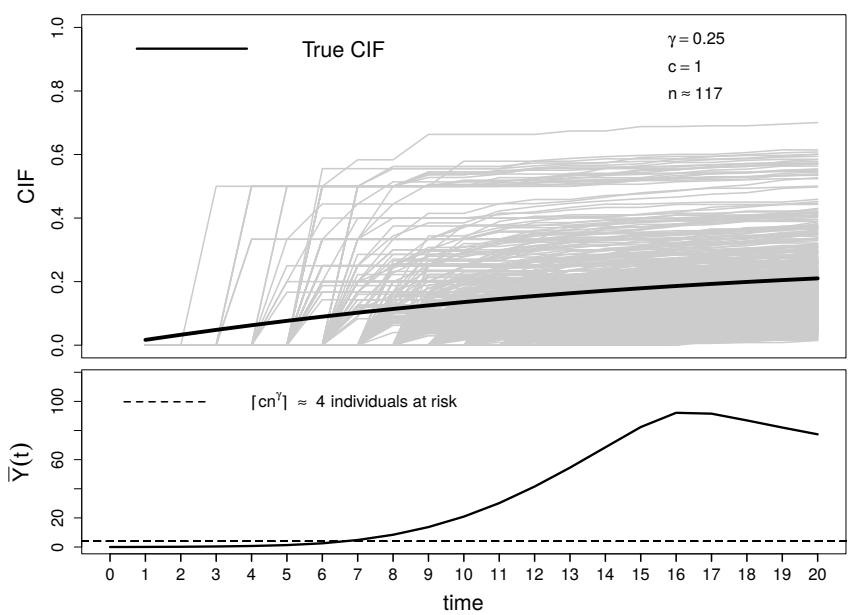

Fig 8: Results of 10000 simulation runs in the small sample scenario: On average $n=117$ out of 200 simulated individuals enter the study. Displayed are true CIF for the event of interest (black line). The gray lines are randomly chosen CIFs estimated using the original Aalen-Johansen estimator. The lower plot shows the average number of individuals at risk over the course of time.

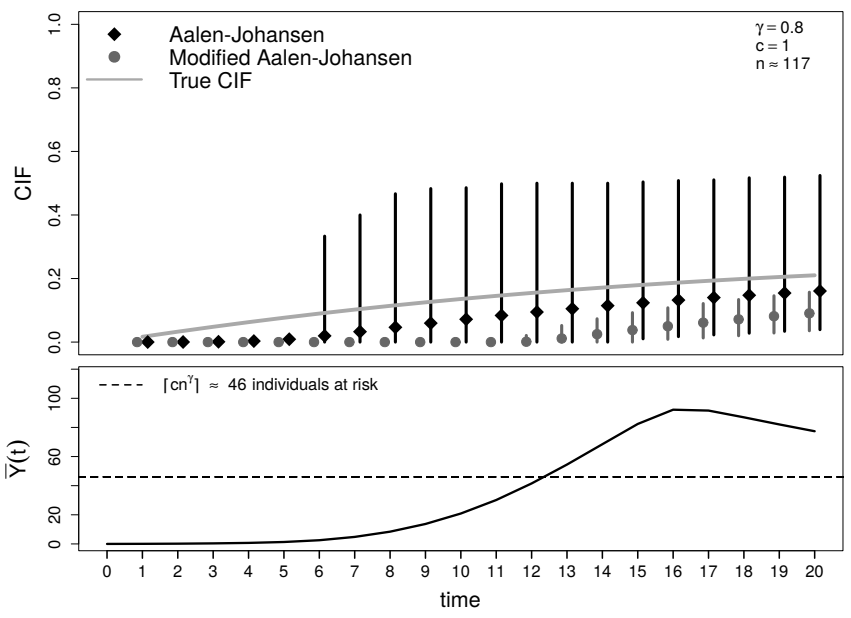

Fig 9: Results of 10000 simulation runs in the small sample scenario: On average $n=117$ out of 200 simulated individuals enter the study. Plot of the mean AalenJohansen estimator and its modified version, along with empirical 95\% CIs. The lower plot shows the average number of individuals at risk over the course of time. The extreme choice of $c$ and $\gamma$ leads to meaningless results. 
9.2. Large sample behavior. For the large sample scenario we simulated $m=10000$ individuals, out of which between 5641 and 6033 individuals entered the study, on average $n=5835$.

We notice that, for a reasonable choice of $\gamma$ and $c$, no difference in the point estimates can be seen and the downward bias from the small sample also disappears asymptotically, see Figure 10 with $\gamma=0.25$ and $c=1$. This shows again that the new estimator is consistent, as long as the border $c n^{\gamma}$ is not too large compared to $n$.

For growing $\mathrm{cn}^{\gamma}$ we observe the same behavior as in the small sample case: The modified estimator is biased downwards with smaller variation, see Figure 11.

We also note that for growing sample sizes, the ratio between estimated and empirical variances tends to one, i.e., estimated and empirical variances essentially become one as sample size grows. This behavior is more pronounced for the modified estimator, see Figure 12.

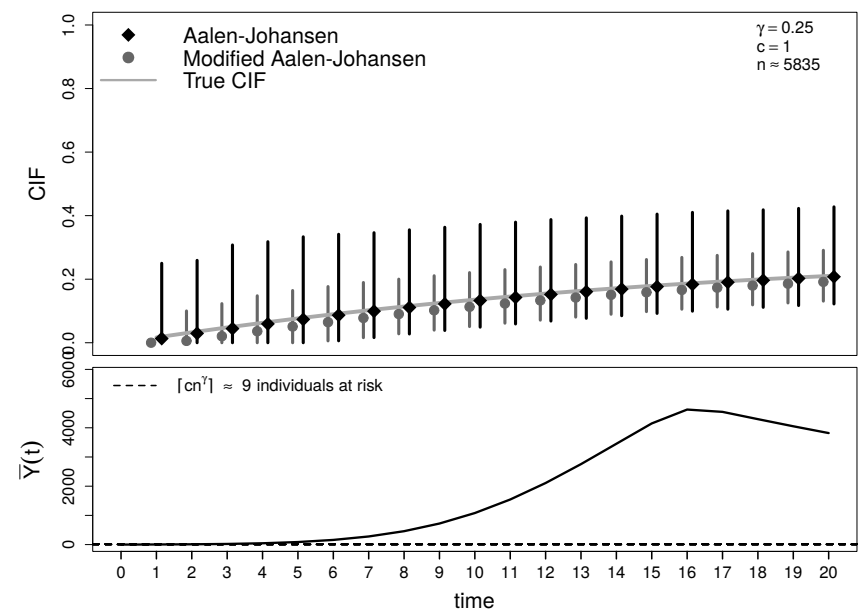

Fig 10: Results of 10000 simulation runs in the large sample setting: On average $n=5835$ out of 10000 simulated individuals enter the study. Displayed are the mean estimators with empirical $95 \%$ CIs and the true CIF. The lower plot shows the average number of individuals at risk.

Tables 5 and 6 contain some results from the simulation study in tabular form. Reported - for both the small sample scenario with $m=200$ simulated individuals and the large sample scenario with $m=10000$ simulated individuals - are the average number of individuals at risk $\bar{Y}(t)$, the bias and relative bias for both the true Aalen-Johansen estimator (AJ) and the modified version (LY), the root mean squared errors (RMSE) for both esti- 


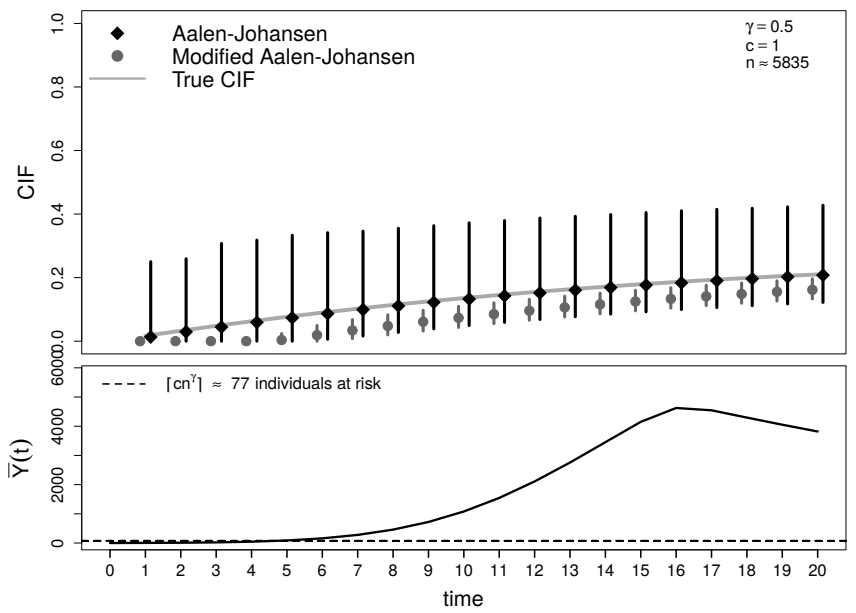

Fig 11: Results of 10000 simulation runs in the large sample setting: On average $n=5835$ out of 10000 simulated individuals enter the study. Displayed are the mean estimators with empirical $95 \%$ CIs and the true CIF. A larger choice of cn $\gamma$ leads to the same behavior as observed for the small sample scenario. The lower plot shows the average number of individuals at risk.

mators as well as the variance estimators. The relative bias is computed as $((\overline{\hat{F}}-F) / F)$ and the RMSE is $\sqrt{(\overline{\hat{F}}-F)^{2}+\operatorname{var}(\hat{F})}$, where $F$ denotes the true CIF for the event of interest and $\overline{\hat{F}}$ is the averaged CIF estimated by either the Aalen-Johansen estimator or the modified estimator.

Note that while both RMSEs decrease to zero as $n$ grows, the distance between them is larger in the large sample behavior. This is due to the fact that the convergence of the RMSE depends on the convergence of both the bias and the empirical variance, which seem to differ between the estimators. For even larger sample sizes, however, the distance between the RMSEs decreases again (results not shown). 

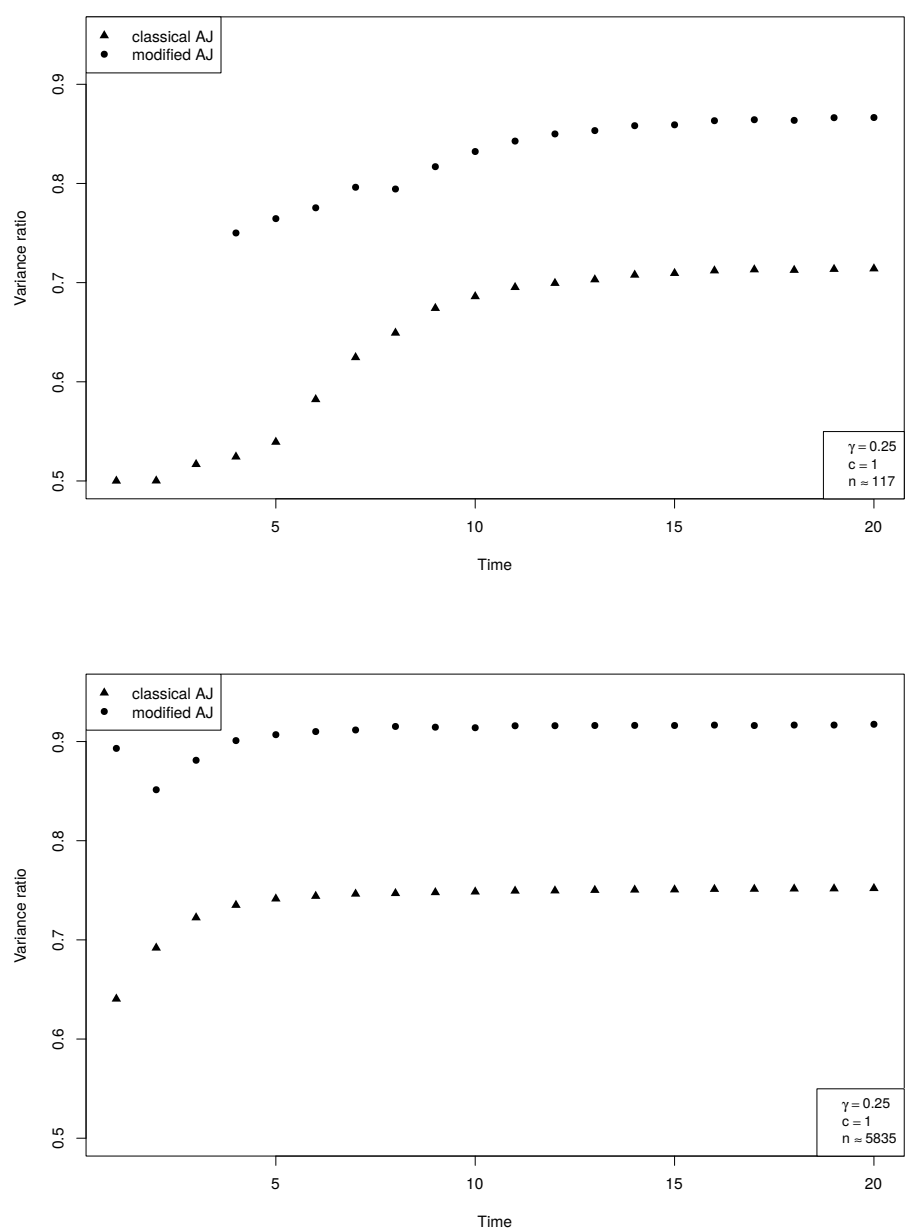

Fig 12: Results of 10000 simulation runs in the small (upper panel) and large (lower panel) sample scenario: Displayed are the ratios between the estimated and the empirical variances of the Aalen-Johansen estimator and its modified version, respectively.

9.3. A cross-validated choice of the tuning parameters. Figure 13 shows the mean estimators for the cross-validated choice of $\left(c_{0}, \gamma_{0}\right)$ from Section 4.3. The results are similar to the ones obtained previously.

In an ideal world where one would have one data set for tuning the parameters and one for evaluation of the data, another method of cross-validation would be feasible. This procedure is described in the following. Again, we restricted our investigation to the small sample simulation setting, where we 


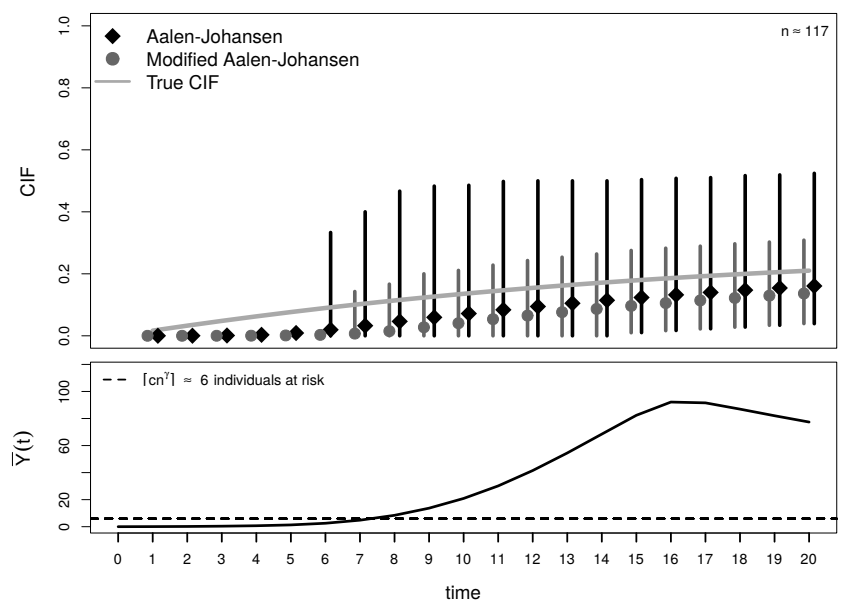

Fig 13: Results of 10000 simulation runs in the cross-validation study: Displayed are the mean Aalen-Johansen estimator and its modification using $\left(c_{0}, \gamma_{0}\right)$ along with $95 \%$ empirical CIs and the number of individuals at risk over the course of time (lower plot). On average $n=117$ out of 200 simulated individuals enter the study.

simulated $2 \times n$ sim data sets for $n$ sim $=10000$ as before. For each pair of data, we obtained a cross-validated choice of the tuning parameters $\tilde{c}_{0}$ and $\tilde{\gamma}_{0}$ in one data set. Using $\tilde{c}_{0}$ and $\tilde{\gamma}_{0}$, the modified Aalen-Johansen estimator was then calculated in the second data set. Average results from the second data set of the 10000 pairs of data sets are reported in Figure 14.

Following Hastie, Tibshirani and Friedman (2011), the cross-validation procedure was conducted as follows: First, we split the data set into $K=5$ approximately equally sized parts. For each part $k \in\{1, \ldots, K\}$, we calculated the modified Aalen-Johansen estimator $\hat{\mathbf{P}}^{(-k)}$ with parameters $(c, \gamma)$ using only the other $K-1$ parts of the data. Following Section 3.3, we then predicted the pregnancy outcome for each individual in part $k$ by

$$
\pi_{i}^{(k)}=\frac{\hat{P}^{(-k)}\left(T>l_{i}, T \leq \infty, X(T)=j\right)}{\hat{S}^{(-k)}\left(T>l_{i}\right)}
$$

where $l_{i}$ denotes the left-truncation time of individual $i$.

The predicted outcomes were compared to the true pregnancy outcomes by estimating the Brier-Score in data part $k$ and as in Section 3.3, $B S^{(k)}=$ $\frac{1}{n_{k}} \sum_{i=1}^{n_{k}}\left(\mathbb{1}\left(X\left(T_{i}\right)=j\right)-\pi_{i}^{(k)}\right)^{2}$, where $n_{k}$ is the number of individuals in 
part $k$. Finally, we averaged over all parts, resulting in

$$
\overline{B S}=\frac{1}{K} \sum_{k=1}^{K} B S^{(k)} .
$$

These steps were conducted for all parameters $(c, \gamma)$ displayed in Table 1 in Section 4.3. The parameters $\left(\tilde{c}_{0}, \tilde{\gamma}_{0}\right)$ resulting in the smallest $\overline{B S}$ were used for the calculation of the results on the second data set.

Combining all 10000 simulation runs resulted in a median value of $\left\lceil c n^{\gamma}\right\rceil \approx$ 5. Figure 14 illustrates findings similar to Section 4.2 for the original choice of $\gamma=0.25$ and $c=1$. We also note that the difference between the two cross-validation approaches is minimal.

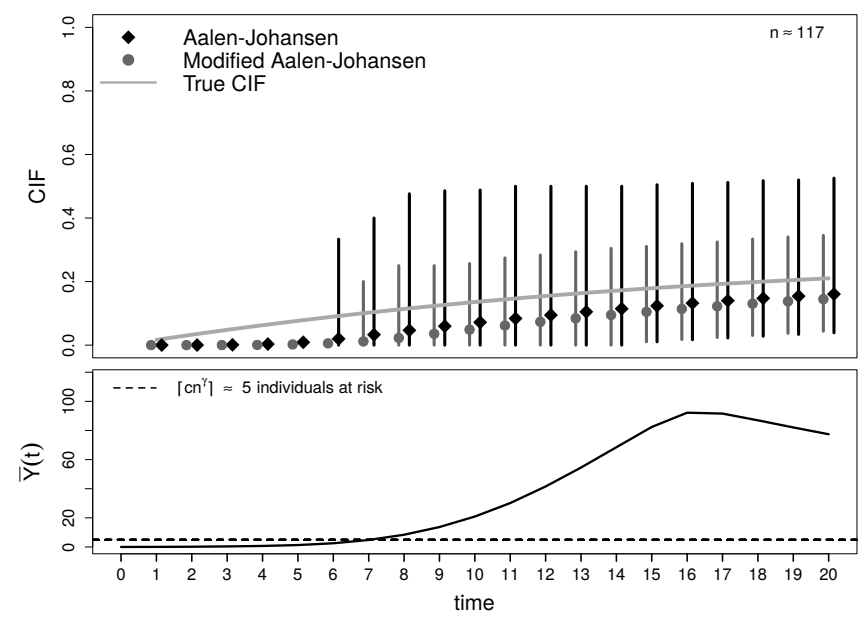

Fig 14: Results of 10000 simulation runs in the cross-validation study: Displayed are the mean Aalen-Johansen estimator and its modification using $\left(\tilde{c}_{0}, \tilde{\gamma}_{0}\right)$ along with $95 \%$ empirical CIs and the number of individuals at risk over the course of time (lower plot). On average $n=117$ out of 200 simulated individuals enter the study.

9.4. Simulation Scenario II. In this scenario, we simulated competing risks data with two competing events again, similar to Allignol, Schumacher and Beyersmann (2010). We chose a constant cause-specific hazard $\alpha_{01}(t)=$ 0.015 for the event of interest and again a Weibull-type hazard for the competing event, $\alpha_{02}(t)=10.5 / 39.5 \cdot(t / 39.5)^{9.5}$. Left-truncation times were simulated independent of $(T, X(T))$, following a gamma distribution $t^{\beta-1} e^{-t / \sigma} / \sigma^{\beta} \Gamma(\beta)$. The parameters $(\beta, \sigma)$ of the gamma distribution were chosen as follows: 
- $(\beta, \sigma)=(2,3)$ for light truncation

- $(\beta, \sigma)=(3.3,3.4)$ for medium truncation

- $(\beta, \sigma)=(7.3,4.3)$ for heavy truncation

Note that the truncation distribution is less extreme than in Scenario I in the sense that more individuals enter the study at early time points. By choosing three different truncation settings, we are also able to better analyze the influence of the truncation distribution on the results.

Again, $m=200$ individuals were simulated. This led on average to $n=$ 184 individuals under study for light truncation, $n=170$ for medium and $n=99$ for heavy truncation. The corresponding plots of the Aalen-Johansen estimator and the modified estimator are displayed in Figures 15-17.

We notice that the modified estimator yields good results in this second scenario. Bias and variability depend on the truncation distribution, which again shows that the higher absolute bias of the modified estimator stems from the fact that small risk sets are excluded from the analysis. However, the difference between the usual Aalen-Johansen estimator and its modification is not as pronounced here as it was in the scenario in Section 4.

Coverage probabilities for the three different truncation scenarios are displayed in Table 4 . The coverage is much better for less pronounced truncation scenarios.

TABLE 4

Coverage probabilities in \% for the modified Aalen-Johansen estimator in Simulation Scenario II with $\gamma=0.25, c=1, m=200$ ( $n \approx 184$ for light truncation, $n \approx 170$ for medium truncation and $n \approx 99$ for heavy truncation).

\begin{tabular}{cccc}
\hline time & light & medium & heavy \\
\hline 1 & 0.79 & 0.00 & 0.00 \\
2 & 23.21 & 0.12 & 0.00 \\
3 & 56.32 & 5.36 & 0.00 \\
4 & 82.17 & 19.03 & 0.00 \\
5 & 93.09 & 40.31 & 0.01 \\
10 & 90.88 & 85.67 & 11.42 \\
15 & 91.58 & 84.79 & 62.40 \\
20 & 92.02 & 85.44 & 79.86 \\
25 & 92.48 & 86.27 & 77.19 \\
30 & 93.33 & 87.70 & 76.24 \\
35 & 93.87 & 88.92 & 76.54 \\
40 & 93.95 & 89.75 & 76.83 \\
45 & 81.28 & 77.44 & 62.37 \\
\hline
\end{tabular}




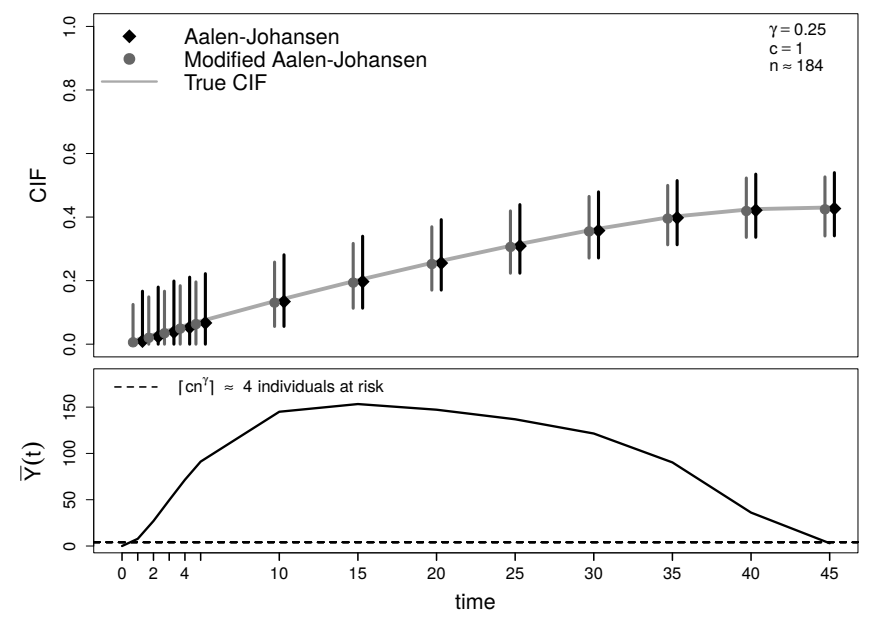

Fig 15: Results of 10000 simulation runs in the second scenario: Displayed are the mean Aalen-Johansen estimator and its modification under light left-truncation along with $95 \%$ empirical CIs and the number of individuals at risk over the course of time (lower plot). On average $n=184$ out of 200 simulated individuals enter the study.

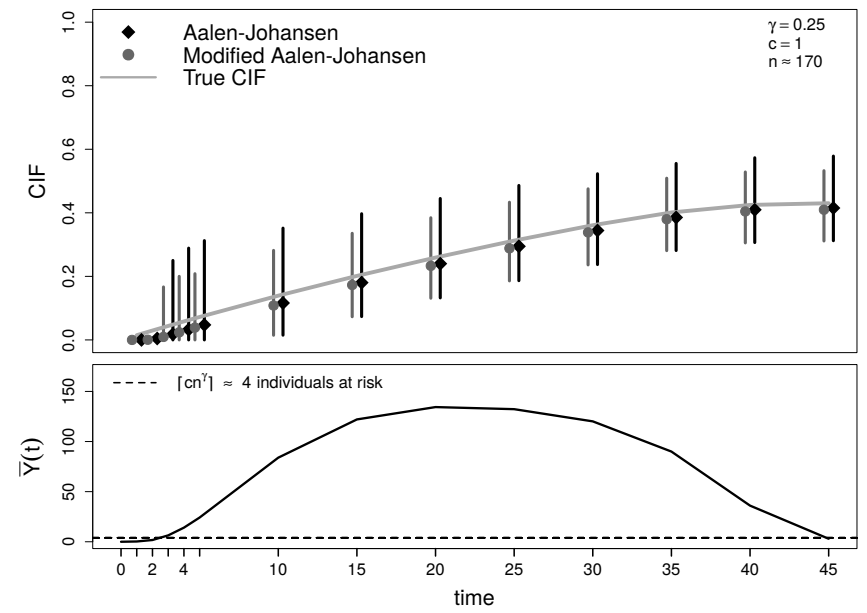

Fig 16: Results of 10000 simulation runs in the second scenario: Displayed are the mean Aalen-Johansen estimator and its modification for medium left-truncation along with $95 \%$ empirical CIs and the number of individuals at risk over the course of time (lower plot). On average $n=170$ out of 200 simulated individuals enter the study. 


\section{SUPPLEMENT TO: NONPARAMETRIC ESTIMATION OF PREGNANCY OUTCOMES}

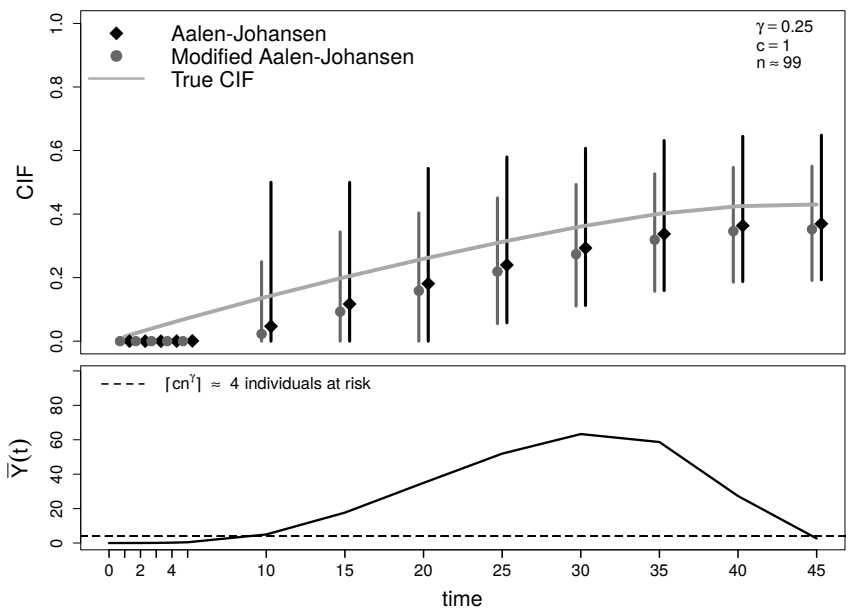

Fig 17: Results of 10000 simulation runs in the second scenario: Displayed are the mean Aalen-Johansen estimator and its modification for heavy left-truncation along with $95 \%$ empirical CIs and the number of individuals at risk over the course of time (lower plot). On average $n=99$ out of 200 simulated individuals enter the study.

\section{References.}

Allignol, A., Schumacher, M. and Beyersmann, J. (2010). A Note on Variance Estimation of the Aalen-Johansen Estimator of the Cumulative Incidence Function in Competing Risks, with a View towards Left-Truncated Data. Biometrical Journal 52 $126-137$.

Andersen, P. K., Borgan, Ø., Gill, R. D. and Keiding, N. (1993). Statistical Models Based on Counting Processes. Springer-Verlag.

Gill, R. D. and Johansen, S. (1990). A survey of product-integration with a view toward application in survival analysis. The Annals of Statistics 18 1501-1555.

Hastie, T., Tibshirani, R. and Friedman, J. (2011). The Elements of Statistical Learning, 2nd ed. Springer.

LAI, T. L. and Ying, Z. (1991). Estimating a distribution function with truncated and censored data. The Annals of Statistics 19 417-442.

Institute of Statistics

Ulm UNIVERSITY, ULM

Germany

E-MAIL: sarah.friedrich@uni-ulm.de 


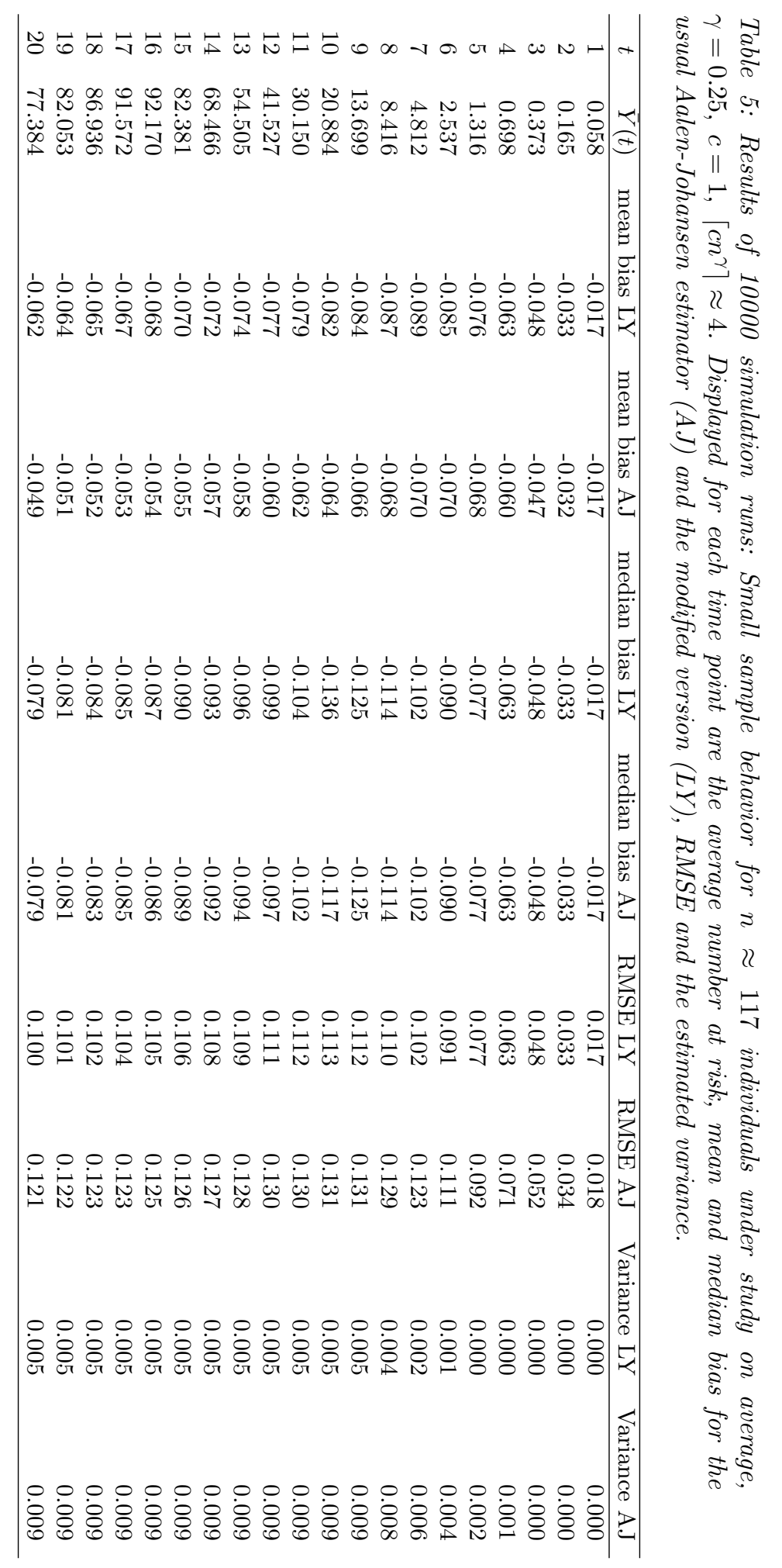




\section{SUPPLEMENT TO: NONPARAMETRIC ESTIMATION OF PREGNANCY OUTCOMES}

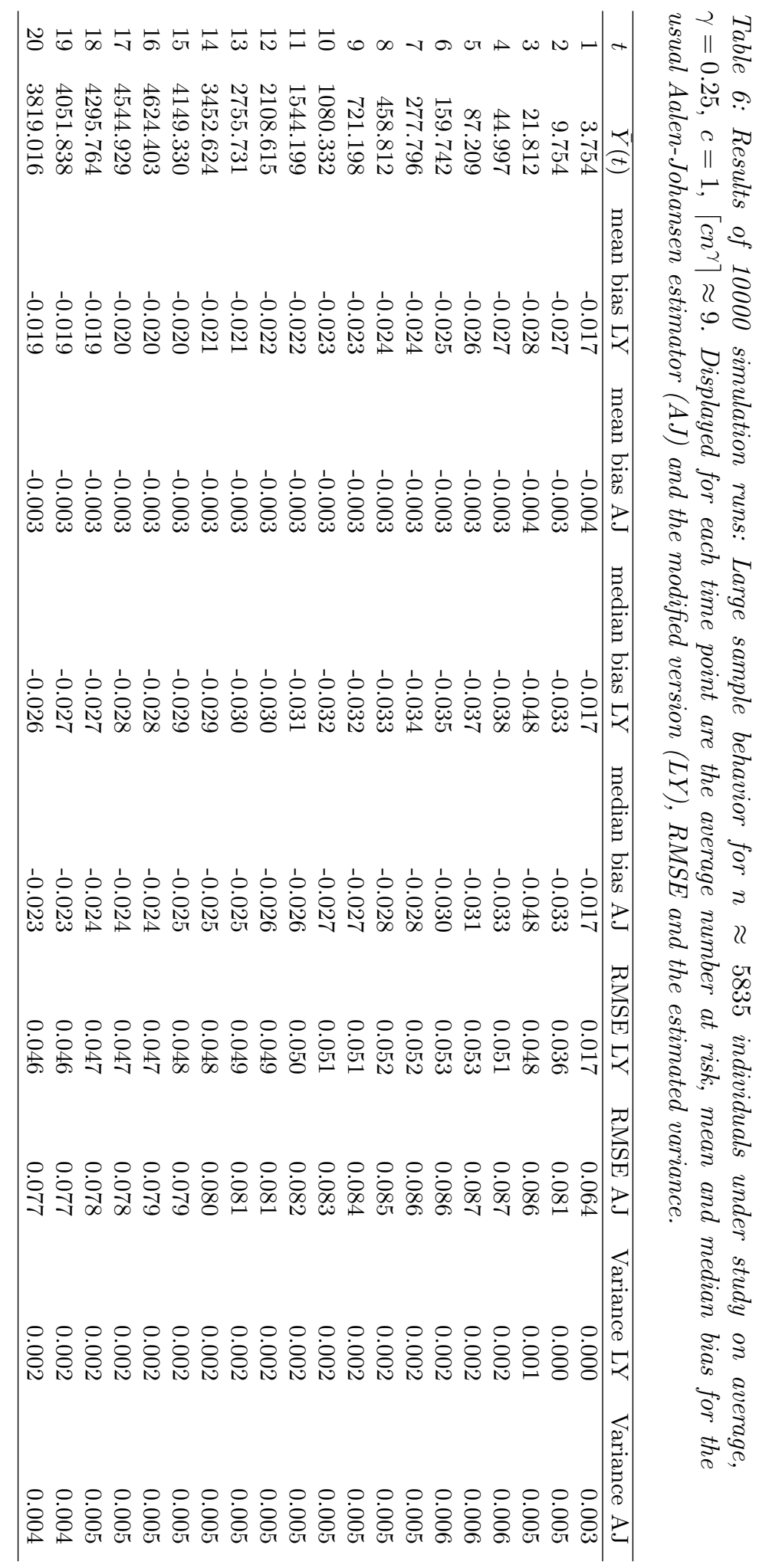

